\title{
Ion Irradiation-Induced Easy-Cone Anisotropy in Double-MgO Free Layers for Perpendicular Magnetic Tunnel Junctions
}

\author{
B. M. S. Teixeira ${ }^{1}$, A. A. Timopheev², N. F. F. Caçoilo', S. Auffret², R. C. \\ Sousa ${ }^{2}$, B. Dieny², E. Alves ${ }^{3}$, N. A. Sobolev ${ }^{1,4}$ \\ 1) Physics Department \& i3N, University of Aveiro, 3810-193 Aveiro, Portugal \\ 2) Université Grenoble Alpes, CEA, CNRS, Grenoble INP, INAC, SPINTEC, F-38000 Grenoble, \\ France \\ 3) IPFN, Instituto Superior Técnico, Universidade de Lisboa, 2695-066 Bobadela LRS, Portugal \\ 4) National University of Science and Technology "MISiS", 119049 Moscow, Russia
}

\begin{abstract}
We have used the ferromagnetic resonance in the $X$-band $(9.37 \mathrm{GHz})$ to investigate the effect of $400 \mathrm{keV} \mathrm{Ar}$ irradiation on the perpendicular magnetic anisotropy (PMA) and Gilbert damping parameter, $\alpha$, of double-MgO free layers designed for application in perpendicular magnetic tunnel junctions. The samples comprised a $\mathrm{MgO} / \mathrm{Fe}_{72} \mathrm{Co}_{8} \mathrm{~B}_{20} / \mathrm{X}(0.2 \mathrm{~nm}) / \mathrm{Fe}_{72} \mathrm{Co}_{8} \mathrm{~B}_{20} / \mathrm{MgO}$ layer stack, where $\mathrm{X}$ stands for an ultrathin Ta or $\mathrm{W}$ spacer. Samples with two different total FeCoB layer thicknesses, $t_{F C B}=3.0 \mathrm{~nm}$ and $t_{F C B}=2.6 \mathrm{~nm}$, were irradiated with ion fluences ranging from $10^{12} \mathrm{~cm}^{-2}$ to $10^{16} \mathrm{~cm}^{-2}$. The effective first-order PMA field, $B_{k 1}$, decreased nearly linearly with the logarithm of the fluence for both FeCoB thicknesses and spacer elements. The decrease in $\mathrm{Bk}_{\mathrm{k} 1}$, which is likely caused by an ion-induced intermixing at the $\mathrm{FeCoB} / \mathrm{MgO}$ interfaces, resulted in a reorientation of the magnetization of the free layers with $\mathrm{tFCB}_{F C}=2.6 \mathrm{~nm}$, initially exhibiting a perpendicular easy-axis anisotropy. For intermediate fluences, $10^{13} \mathrm{~cm}^{-2}$ and $10^{14} \mathrm{~cm}^{-2}$, easy-cone states with different cone angles could be induced in the free layer with a W spacer. Importantly, no corresponding increase in the Gilbert damping was observed. This study shows that ion irradiation can be used to tune the easy-cone anisotropy in perpendicular magnetic tunnel junctions, which is interesting for spintronic applications such as spin-torque magnetic memories, oscillators and sensors.
\end{abstract}




\section{Introduction}

The interfacial perpendicular magnetic anisotropy (PMA) existing at the $\mathrm{FeCoB} / \mathrm{MgO}$ interface is at the origin of the out-of-plane magnetized magnetic tunnel junctions (pMTJ) which serve as the basic storage elements of spin-transfer-torque magnetic random-access memory (STTMRAM). These memories offer a higher storage density, higher thermal stability and lower power consumption than their in-plane counterparts ${ }^{1}$.

For a sufficiently thin FeCoB film, the PMA overcomes the shape anisotropy, resulting in a perpendicular orientation of the magnetization. To increase the free(storage)-layer volume, $\mathrm{V}$, and thus to improve the data retention, while keeping a strong PMA, two $\mathrm{FeCoB} / \mathrm{MgO}$ interfaces may be used ${ }^{1}$. To absorb the boron out of the $\mathrm{FeCoB}$ layers upon the post-deposition anneals required to recrystallize the $\mathrm{MgO}$ barrier and the $\mathrm{FeCoB}$ ferromagnetic (FM) electrodes, a thin metal (e.g., $\mathrm{Ta}, \mathrm{W})$ spacer is usually introduced in the middle of the $\mathrm{FeCoB}$ storage layer ${ }^{2-7}$. Besides improving the data retention, the double-MgO storage layer also exhibits a reduced Gilbert damping, $\alpha$, as compared to thinner ones sandwiched between a single MgO barrier and a heavymetal layer such as Ta or W. In the latter structures, a damping enhancement is often observed resulting from the spin-pumping effect. The suppression of spin pumping in the double-MgO free layers can result in a more than twofold decrease of the damping (and therefore of the critical current for switching, $I_{c 0}$ ) accompanied by an almost doubling of PMA (and consequently, of the thermal stability factor, $\Delta=\mathrm{K}_{\text {eff }} \mathrm{V} / \mathrm{k}_{\mathrm{B}} \mathrm{T}$, where $\mathrm{K}_{\mathrm{eff}}$ is an effective PMA, $\mathrm{k}_{\mathrm{B}}$ the Boltzmann constant and $\mathrm{T}$ the absolute temperature). The result is an overall improved switching efficiency, $\Delta / \mathrm{l}_{\mathrm{c}}$, which further increases as the pMTJ dimensions are reduced ${ }^{3,8}$. Beside free layers for STTMRAM, double-MgO free layers with a Ta spacer have also been proposed for the development of synthetic ferrimagnetic bilayers ${ }^{9}$ as well as remnant spin injectors onto GaAs-based light emitting diodes ${ }^{10}$.

One issue associated with pMTJ stacks is the stochasticity of the STT switching, as a misalignment between the fixed- and free-layer magnetization is necessary for a transfer of angular momentum by a spin-polarized current to occur. Such a misalignment is introduced by thermal fluctuations, but these cause a broad distribution of the switching times due to their random nature. As shown by analytical calculations ${ }^{11}$ and macrospin simulations ${ }^{12}$, setting the free layer in a magnetic easy-cone state, with the cone angle providing the misalignment, would greatly improve the switching characteristics of the pMTJ. Such an easy-cone state is described by phenomenologically including a second-order term, $\mathrm{K}_{2}$, in the PMA energy density of a thin film, in addition to the first-order term, $K_{1}$, i.e., $U_{P M A}=-K_{1} \cos ^{2} \theta-K_{2} \cos ^{4} \theta$, with $\theta$ being the angle between the magnetization and the normal to the film plane. An easy-cone equilibrium state emerges when $\mathrm{K}_{1}>0, \mathrm{~K}_{2}<0$ and $-\mathrm{K}_{2} / \mathrm{K}_{1}>0.5$. Beside certain alloys (e.g., NdCo5 (Ref. ${ }^{13}$ ) and $\mathrm{Mn}_{2} \mathrm{RhSn}\left(\right.$ Ref. $\left.{ }^{10}\right)$ ), multi-layers containing the $\mathrm{FeCoB} / \mathrm{MgO}$ interface can also exhibit easy-cone anisotropy, when the FeCoB thickness is properly adjusted to be close to the reorientation from out-of-plane to in-plane anisotropy ${ }^{14-19}$. 
It has been analytically shown that a negative $\mathrm{K}_{2}$ can arise from spatial fluctuations of $\mathrm{K}_{1}$ (Ref. ${ }^{20}$ ). In our previous study on granular $\mathrm{MgO} / \mathrm{FeCoB} / \mathrm{Ta}$ (Ref. ${ }^{17}$ ) the micromagnetic origin of $\mathrm{K}_{2}$ was demonstrated and it was shown that its magnitude was determined by the ratio between the thickness-dependent magnetic inhomogeneities, such as spatial fluctuations of $K_{1}$, and the intergrain exchange coupling. Another approach to explain the origin of $\mathrm{K}_{2}$, proposed by J. Sun ${ }^{21}$, considers the simultaneous presence of two effects: weakening of the exchange near the $\mathrm{MgO} / \mathrm{FM}$ interface and a strong interface-concentrated PMA efficiently acting only on the very first atomic layers of the magnetic film. In this case, the resulting 'exchange-spring'-like effect can also be at the origin of a negative $\mathrm{K}_{2}$.

One should notice that the easy-cone anisotropy can be useful not only for STT-MRAM but also for other spintronic applications such as spin-torque oscillators ${ }^{22}$ and magnetic sensors ${ }^{11,19}$. However, reproducibly controlling the easy-cone anisotropy in $\mathrm{FeCoB} / \mathrm{MgO}$ systems may be technologically challenging, as this state is only found within a narrow range of layer thicknesses. It would thus be desirable to find a post-deposition process for reorienting the magnetization direction via the control of the interfacial PMA. In that regard, high-energy particle irradiation appears as a simple and reproducible tool to physically modify the interfaces and thus control interfacial magnetic phenomena. A review of the work published in this field until 2004 is given in Ref. ${ }^{23}$. Light ion $\left(\mathrm{He}^{+}\right)$irradiation has been used to reduce the PMA in $\mathrm{Pt} / \mathrm{Co}$ multilayers by promoting local intermixing at the interfaces ${ }^{24}$ and thus to manipulate the magnetization direction of such systems, from out-of-plane to in-plane and also to oblique orientations ${ }^{25}$. Heavier ions such as $\mathrm{Ar}^{+}$were used to modify the properties of $\mathrm{Pt} / \mathrm{Co}$ films as well ${ }^{26}$. More recently, $\mathrm{He}^{+}$ irradiation was used to manipulate the direction of the exchange-bias field in MTJ stacks ${ }^{27}$, to reduce the annealing temperature for crystallizing CoFeB in MgO-based MTJs ${ }^{28}$, and to control the domain wall velocity ${ }^{29}$. Here, we explore the possibility of inducing an easy-cone anisotropy in the technologically relevant $\mathrm{MgO} / \mathrm{FeCoB} / \mathrm{MgO}$ free layers, with and without inclusion of $\mathrm{Ta}$ or W spacers, by $400 \mathrm{keV} \mathrm{Ar}{ }^{+}$irradiation.

\section{Experimental details}

Free layers composed of $\mathrm{Ta}(3) / \mathrm{Ru}(2) / \mathrm{W}(3) / \mathrm{Ta}(1) / \mathrm{FeCoB}(0.3) / \mathrm{MgO}(1.3) / \mathrm{FeCoB}(1.4)$ / $\mathrm{X}(0.2) / \mathrm{FeCoB}(\mathrm{t}) / \mathrm{MgO}(1.1) / \mathrm{W}(2) / \mathrm{Pt}(3)$, where $\mathrm{X}$ stands for a Ta or W spacer, and FeCoB is a $\mathrm{Fe}_{72} \mathrm{C}_{8} \mathrm{~B}_{20}$ alloy, were deposited by magnetron sputtering onto a thermally oxidized 4-inch $\mathrm{Si}$ wafer and annealed at $300^{\circ} \mathrm{C}$ for $2 \mathrm{~min}$. The numbers in parentheses are nominal thicknesses in nanometers. The total thickness of the $\mathrm{FeCoB}$ free layer $\left(\mathrm{t}_{\mathrm{FCB}}=1.4+\mathrm{tnm}\right)$ was varied by growing the topmost FeCoB layer as a wedge $(0.4<t<1.6 \mathrm{~nm})$. Free layers with equal thickness but without spacer were also prepared. Details regarding the sample preparation can be found in Ref. ${ }^{30}$ for similarly prepared structures.

Samples with an area of $4 \times 4 \mathrm{~mm}^{2}$ were cut from the wafer ensuring a negligible $( \pm 0.18 \AA)$ nominal thickness gradient. Two thicknesses of the $\mathrm{FeCoB}$ layer were selected for two irradiation runs: $t_{F C B}=3.0 \mathrm{~nm}$ and $t_{F C B}=2.6 \mathrm{~nm}$. The irradiation with $400 \mathrm{keV} \mathrm{Ar}+$ ions was performed at five 
different fluences, $\Phi$, ranging from $10^{12} \mathrm{~cm}^{-2}$ to $10^{16} \mathrm{~cm}^{-2}$. The irradiation conditions guarantee that elastic and inelastic interactions occur within the multilayers, with expected intermixing of elements across the interfaces (changes in the element concentration profiles smaller than $7 \times 10^{-}$ 14 in units of $\% \Phi^{-1}$ ), whereas the $\mathrm{Ar}^{+}$ions are deposited inside the Si substrate (not shown), as simulated by the TRIM (Transport of lons in Matter) package included in the SRIM software ${ }^{31}$.

The magnetic properties of the free layers were investigated using angle-dependent ferromagnetic resonance (FMR) measurements performed at room temperature at a microwave frequency of $9.37 \mathrm{GHz}$ in an EPR spectrometer. To account for small variability of magnetic properties of the free layers cut from the same wafer, the FMR spectra were acquired for all samples before and after irradiation. From the out-of-plane angular dependence of the resonance field, $B_{r e s}$ (see, e.g., figure 3), we estimated the first- and second-order anisotropy contributions, starting from the description of the magnetic free-energy density:

$$
\frac{\mathrm{U}}{\mathrm{M}_{\mathrm{S}}}=-\mathrm{B} \cos \left(\theta_{\mathrm{B}}-\theta_{\mathrm{M}}\right)-\frac{1}{2} \mathrm{~B}_{\mathrm{K} 1} \cos ^{2} \theta_{\mathrm{M}}-\frac{1}{4} \mathrm{~B}_{\mathrm{K} 2} \cos ^{4} \theta_{\mathrm{M}},
$$

where $B_{K 1}=\left(2 k_{s 1} /\left(t_{F C B} M_{S}\right)\right)-4 \pi M_{S}$ is the effective first-order anisotropy field resulting from the competing contributions of the interfacial PMA $\left(2 \mathrm{k}_{\mathrm{s} 1} / \mathrm{t}_{\mathrm{FCB}}\right)$ and the thin-film shape anisotropy $\left(4 \pi \mathrm{Ms}^{2}\right) ; B_{\mathrm{K} 2}=4 \mathrm{~K}_{2} / \mathrm{M}_{\mathrm{S}}$ is the effective second-order anisotropy field; $k_{s 1}$ is the first-order interfacial PMA constant; $K_{2}$ the second-order volume PMA constant; $M s$ the saturation magnetization, and $\theta_{M}\left(\theta_{B}\right)$ the angle between the magnetization (external field) and the film normal. For details of the general development of the model to extract the FMR modes, $\omega(B)$, see, e.g., Refs. ${ }^{13,32}$.

\section{Results}

The 3.0-nm-thick FeCoB layers originally exhibited an easy-plane anisotropy, while the 2.6$\mathrm{nm}$-thick layers with a Ta or W spacer were initially in the easy-axis regime, with the magnetization oriented along the film normal. Table I summarizes the mean values and standard deviations of the anisotropy fields extracted before irradiation, $\mathrm{B}_{\mathrm{K} 1,2}^{0}$, for the different spacer and film thickness configurations. After irradiation, the anisotropy fields, $\mathrm{B}_{\mathrm{Ki}}^{\mathrm{F}}$, were extracted for each ion fluence, and the variations of $\mathrm{B}_{\mathrm{Ki}}$ were calculated as $\delta\left(\mathrm{B}_{\mathrm{Ki}}\right)=\mathrm{B}_{\mathrm{Ki}}^{\mathrm{F}}-\mathrm{B}_{\mathrm{Ki}}^{0}, \mathrm{i}=1,2$ (figure 1).

After irradiation, the FMR line shifted toward higher (lower) fields for out-of-plane (in-plane) applied magnetic field. Interestingly, as the line shifted, additional weaker resonances became evident (not shown). Up to this moment we have not developed a physical understanding of those additional lines and limited the following analysis to the main FMR line (i.e. the line evident both before and after irradiation). 
Table I: Mean first- and second-order anisotropy fields extracted before irradiation.

\begin{tabular}{ccc}
\hline Free layer & $\mathrm{B}_{\mathrm{K} 1}^{\mathbf{0}}(\mathbf{k G})$ & $\mathrm{B}_{\mathrm{K} 2}^{\mathbf{0}}(\mathbf{k G})$ \\
\hline $\mathrm{MgO} / \mathrm{FeCoB}(3.0) / \mathrm{MgO}$ & $-4.098 \pm 0.046$ & $-0.279 \pm 0.016$ \\
$\mathrm{MgO} / \mathrm{FeCoB}(1.4) / \mathrm{W}(\mathbf{0 . 2}) / \mathrm{FeCoB}(1.6) / \mathrm{MgO}$ & $-0.402 \pm 0.036$ & $-0.689 \pm 0.004$ \\
$\mathrm{MgO} / \mathrm{FeCoB}(\mathbf{1 . 4}) / \mathrm{Ta}(\mathbf{0 . 2}) / \mathrm{FeCoB}(1.6) / \mathrm{MgO}$ & $-0.865 \pm 0.058$ & $-0.492 \pm 0.003$ \\
$\mathrm{MgO} / \mathrm{FeCoB}(1.4) / \mathrm{W}(\mathbf{0 . 2}) / \mathrm{FeCoB}(1.2) / \mathrm{MgO}$ & $0.939 \pm 0.125$ & $-0.424 \pm 0.016$ \\
$\mathrm{MgO} / \mathrm{FeCoB}(\mathbf{1 . 4}) / \mathrm{Ta}(\mathbf{0 . 2}) / \mathrm{FeCoB}(\mathbf{1 . 2}) / \mathrm{MgO}$ & $0.838 \pm 0.040$ & $-0.402 \pm 0.004$ \\
\hline
\end{tabular}

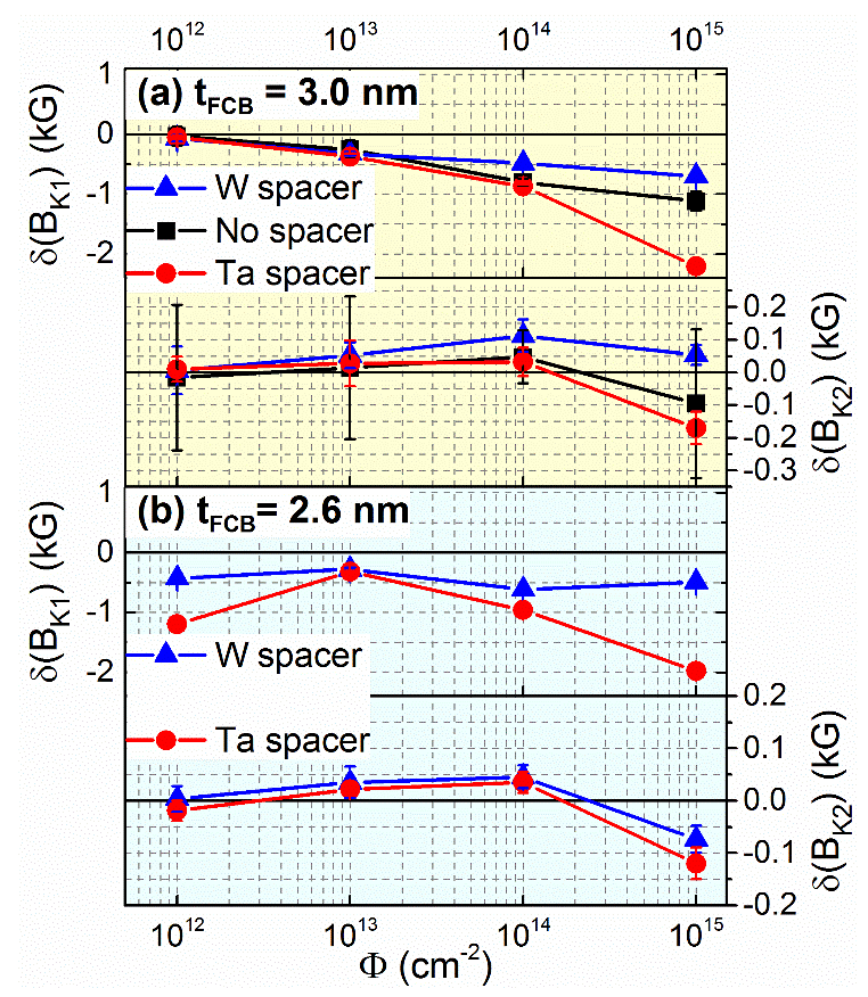

Figure 1: Absolute variation of the first- and second-order anisotropy fields with ion fluence $\left(\delta\left(B_{K i}\right)=B_{K i}^{F}-\right.$ $B_{K i}^{0}$ ) for (a) 3.0-nm-thick and (b) 2.6-nm-thick FeCoB films with a W spacer (triangles), Ta spacer (dots) or no spacer (squares). Table 1 provides the reference values before irradiation, $B_{K i}^{0}$, for each of the layers.

The top panels of figures $1 a$ ) and $1 b$ ) show the nearly linear decrease of $B_{k 1}$ with the logarithm of the ion fluence for $t_{F C B}=3.0 \mathrm{~nm}$ and $t_{F C B}=2.6 \mathrm{~nm}$, respectively. While a fluence of $10^{12} \mathrm{~cm}^{-2}$ produces almost no change in anisotropy for the $3.0-\mathrm{nm}$-thick sample, at $10^{15} \mathrm{~cm}^{-2}$ the $B_{k 1}$ value decreases significantly in the free layers with the $\mathrm{W}$ spacer, no-spacer and Ta spacer by about $-0.7 \mathrm{kG},-1.1 \mathrm{kG}$ and $-2.2 \mathrm{kG}$, respectively. In the $2.6 \mathrm{~nm}$-thick free layer, a decrease of $\mathrm{B}_{\mathrm{k} 1}$ also occurs (the $10^{12} \mathrm{~cm}^{-2}$ point is treated as an outlier, and the value for $10^{15} \mathrm{~cm}^{-2}$ and the $\mathrm{W}$ spacer may be explained by an increased inhomogeneity of the initial sample inherent to these thinner layers). Both irradiation runs showed a higher decrease in $B_{K 1}$ when the Ta spacer was 
used instead of the $\mathrm{W}$ one. A slight decrease of $\left|\mathrm{B}_{\mathrm{k} 2}\right|$ with the ion fluence increasing up to $10^{14}$ $\mathrm{cm}^{-2}$ was also observed (bottom panels of figures $1 \mathrm{a}$ ) and $1 \mathrm{~b}$ )). At $10^{16} \mathrm{~cm}^{-2}$ the FMR line disappeared in all cases, probably due to a strong sample intermixing inducing a ferromagneticparamagnetic transition. Both the nearly unchanged $B_{k 1}$ for $\Phi=10^{12} \mathrm{~cm}^{-2}$ and the lack of FMR at $\Phi=10^{16} \mathrm{~cm}^{-2}$ are consistent with the order of magnitude found in the TRIM simulations for the maximum profile concentration changes (around $7 \times 10^{-14} \% \Phi^{-1}$ ).

Looking at the definition of $\mathrm{B}_{\mathrm{K} 1}$, its decrease can be explained either by an increase of $\mathrm{Ms}_{\mathrm{s}}$ or by a decrease of $k_{s 1}$. Reports on Co thin films $(0.5 \mathrm{~nm})$ have shown $M s$ to decrease upon a $10^{16}$ $\mathrm{cm}^{-2} \mathrm{He}^{+}$irradiation at $30 \mathrm{keV}$ (Ref. ${ }^{25}$ ). In Ref. ${ }^{33}$, the authors reported an unchanged Ms of $\mathrm{Co} / \mathrm{Pd} / \mathrm{Co} / \mathrm{Ni}$ multilayers irradiated with up to $10^{15} \mathrm{He}^{+} / \mathrm{cm}^{2}$ at $20 \mathrm{keV}$. Assuming $\mathrm{Ms}$ does not increase with the ion irradiation in our case, which is reasonable when accounting for the loss of the magnetic ordering observed for $\Phi=10^{16} \mathrm{~cm}^{-2}$, the reduction of $B_{k 1}$ may be ascribed to a drop of $\mathrm{k}_{\mathrm{s}}$. Like in Ref. ${ }^{25}$, this is consistent with an elemental mixing at the $\mathrm{FeCo} / \mathrm{MgO}$ interface, in our case modifying interfacial $\mathrm{Fe}(\mathrm{Co})-\mathrm{O}$ electronic hybridization at the origin of the PMA and thus resulting in a reduction of $\mathrm{k}_{\mathrm{s} 1}$. Furthermore, heavier ions, such as $\mathrm{Ar}^{+}$, are more efficient in ballisticand defect-enhanced diffusion intermixing at the interfaces than $\mathrm{He}^{+}$, due to a higher energy deposition in elastic collisions. Indeed, for equal energies, lower fluences of $\mathrm{Ar}^{+}$than of $\mathrm{He}^{+}$have been shown to reduce the coercivity by the same amount ${ }^{26}$. Irradiation-induced migration of $B, W$ or Ta to the $\mathrm{FeCoB} / \mathrm{MgO}$ interface or alloying of these elements with Fe may also lead to a local decrease of $k_{s 1}$, as well as to a reduction of Ms.

Several investigations ${ }^{24-26}$ reported similar irradiation-induced modulations of $\mathrm{Bk}_{\mathrm{k}}$ in $\mathrm{Co} / \mathrm{Pt}$ multilayers with interfacial PMA, attributing their origin to interfacial mixing. Here we have shown that the PMA in the $\mathrm{MgO} / \mathrm{FeCoB} / \mathrm{MgO}$ system decreases with increasing $\mathrm{Ar}^{+}$ion fluence. The anisotropy modulation resulted in a reorientation of the magnetization direction: while the 3.0-nmthick free layers were driven deeper into the easy-plane regime, spin reorientations were effectively induced in the free layers with $\mathrm{t}_{\mathrm{FCB}}=2.6 \mathrm{~nm}$, as highlighted in figure 2 . 

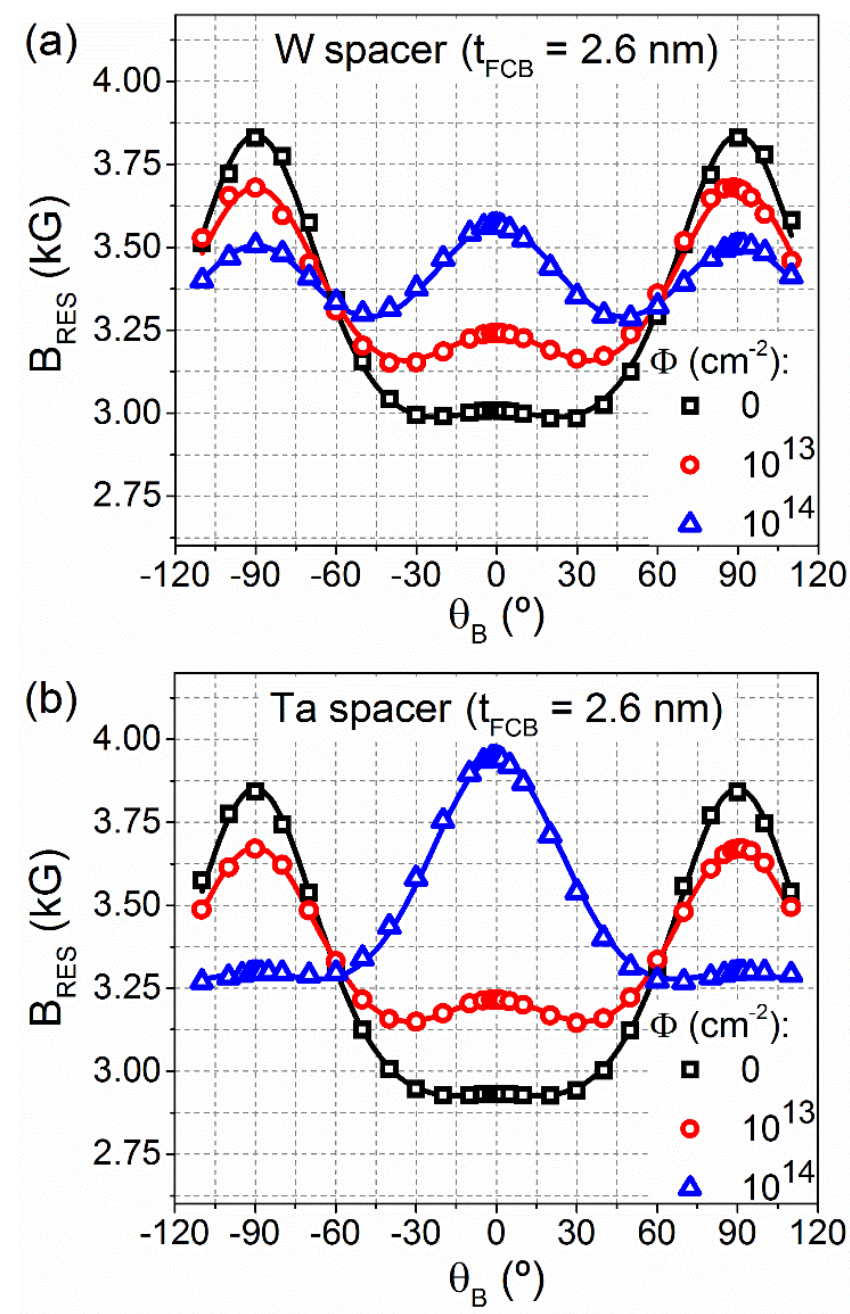

Figure 2: Out-of-plane angular dependences of the resonance field, for $\mathrm{tFCB}_{\mathrm{FC}}=2.6 \mathrm{~nm}$ with a W (a) or Ta (b) spacer for different ion fluences: $0 \mathrm{~cm}^{-2}$ (squares), $10^{13} \mathrm{~cm}^{-2}$ (dots) and $10^{14} \mathrm{~cm}^{-2}$ (triangles). The solid lines are fits to the data.

The free layer with a W spacer initially exhibits a uniaxial perpendicular magnetic anisotropy. After an $\mathrm{Ar}^{+}$irradiation with $10^{13} \mathrm{~cm}^{-2}$ and $10^{14} \mathrm{~cm}^{-2}$, the magnetization is driven into the easycone state with the cone angle increasing to $30^{\circ}$ and $45^{\circ}$, respectively. In the free layer with a $\mathrm{Ta}$ spacer, the stronger decrease of $\mathrm{B}_{\mathrm{K} 1}$ leads to a complete reorientation of the magnetization from easy axis (before irradiation) to easy cone at $10^{13} \mathrm{~cm}^{-2}$ and then to easy plane at $10^{14} \mathrm{~cm}^{-2}$. 

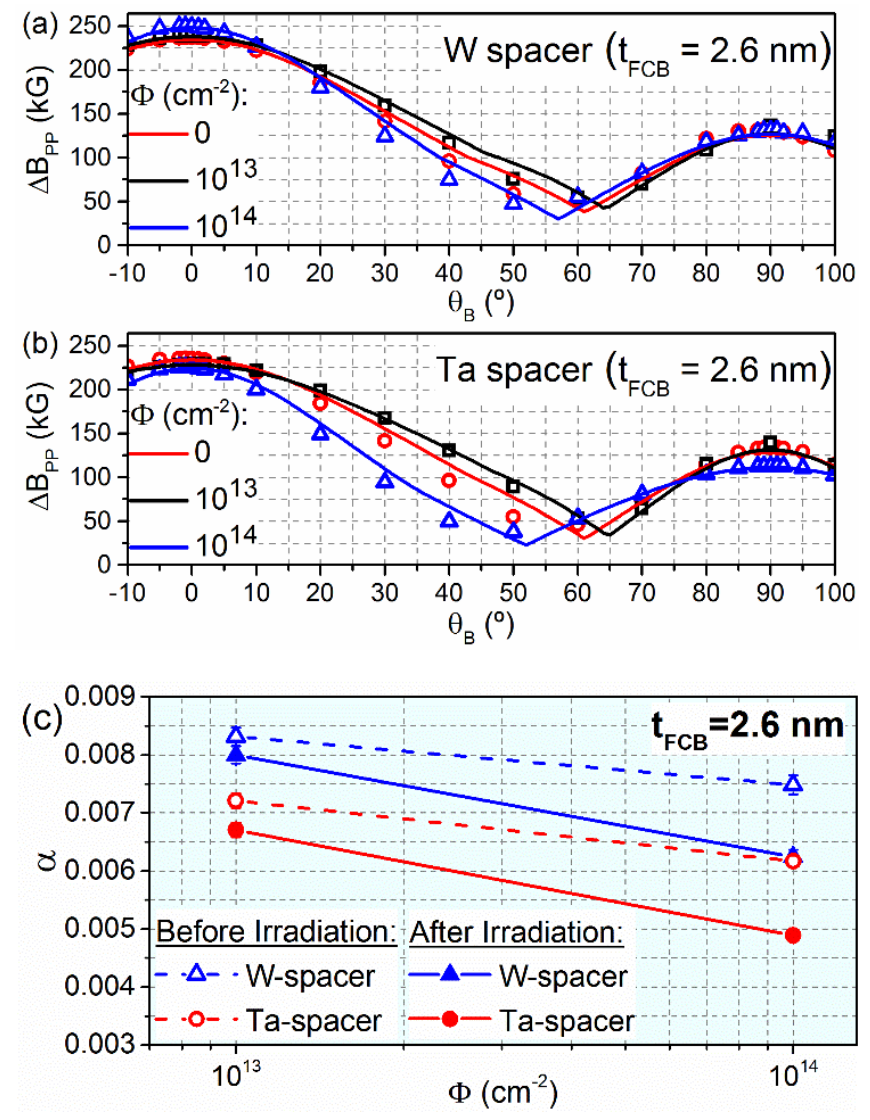

Figure 3: (a-b) Out-of-plane angular dependences of the peak-to-peak linewidth, for tFCB $=2.6 \mathrm{~nm}$ with a W (a) or Ta (b) spacer for different irradiation fluences: $0 \mathrm{~cm}^{-2}$ (squares), $10^{13} \mathrm{~cm}^{-2}$ (dots) and $10^{14} \mathrm{~cm}^{-2}$ (triangles). The solid lines are fits to the data. (c) Gilbert damping parameter extracted before (open symbols and dashed lines) and after (solid symbols and lines) irradiation.

The effect of the irradiation on the peak-to-peak linewidth, $\Delta B P P$, was also assessed for those free layers whose magnetization direction was reoriented (figures $3 a$ ) and $3 b$ )). We modelled the $\Delta \operatorname{BPP}\left(\theta_{M}\right)$ dependence using two contributions to the linewidth: the intrinsic one, proportional to the Gilbert damping, $\alpha$, and the inhomogeneous one caused by fluctuations of the anisotropy fields, $\Delta\left(\mathrm{B}_{\mathrm{Ki}}\right)$. Fluctuations of the anisotropy direction, $\Delta \theta_{\mathrm{B}}$, were found to be negligible in our layers. Following the method of Ref. ${ }^{34}, \Delta \mathrm{B}_{\mathrm{PP}}$ is written as a first-order Taylor series expansion of $\Delta \mathrm{B}_{\mathrm{PP}}^{\mathrm{Ki}}$ and $\Delta \mathrm{B}_{\mathrm{PP}}^{\alpha}$ versus the broadening contributions, $\Delta\left(\mathrm{B}_{\mathrm{Ki}}\right)$ and $\Delta \omega=\alpha \gamma\left(\partial^{2} \mathrm{U} / \partial \theta_{\mathrm{M}}^{2}\right)$, as:

$$
\Delta \mathrm{B}_{\mathrm{PP}}=\frac{1}{\sqrt{3}}\left(\Delta\left(\mathrm{B}_{\mathrm{Ki}}\right)\left|\frac{\partial \mathrm{B}_{\mathrm{RES}}}{\partial \mathrm{B}_{\mathrm{Ki}}}\right|+\alpha \gamma \frac{\partial^{2} \mathrm{U}}{\partial \theta_{\mathrm{M}}^{2}}\left|\frac{\partial \mathrm{B}_{\mathrm{RES}}}{\partial \omega}\right|\right)
$$

where $\gamma$ and $\omega$ are the gyromagnetic ratio and the magnetization precession frequency, respectively.

We found the linewidth to be dominated by the inhomogeneous broadening caused by spatial fluctuations of $B_{K 1}$ : the lines are broader for $\theta_{B}=0^{\circ}$ than for $\theta_{B}=90^{\circ}$, and at an intermediate angle the inhomogeneous contribution is cancelled out (i.e. the $\operatorname{BrES}(\theta)$ dependencies calculated for a fluctuating $\mathrm{B}_{\mathrm{k} 1}$ coincide in one angular point, where $\left.\partial \mathrm{B}_{\mathrm{RES}} / \partial \mathrm{B}_{\mathrm{k} 1}=0\right)$, resulting in narrower lines. 
The intrinsic broadening, on the other hand, has a different angular dependence, with equal minima found at $\theta_{B}=0^{\circ}$ and $\theta_{B}=90^{\circ}$ and can therefore be distinguished from the inhomogeneous one during the fitting. From the fittings of figures $3 a$ ) and $3 b$ ), we made the estimation of a reported in figure 3c).

Compared to the values extracted before irradiation (open symbols and dashed lines in figure 3), $\alpha$ appears to have decreased for the free layers irradiated with fluences of $10^{13} \mathrm{~cm}^{-2}$ and $10^{14} \mathrm{~cm}^{-2}$. These $\alpha$ values, ranging from approximately 0.008 to 0.004 , are quite reasonable considering the thickness of the $\mathrm{FeCoB}$ films in our double-MgO structures. Similar values were obtained in Refs. ${ }^{35,36}$ for $\mathrm{Ta} / \mathrm{CoFeB} / \mathrm{MgO}$ structures with similar thicknesses of the CoFeB layer.

Summarizing, we have shown that $\mathrm{Ar}^{+}$irradiation can induce an easy-cone state in $\mathrm{MgO} / \mathrm{FeCoB} / \mathrm{MgO}$ free layers with metallic spacers, without increasing the Gilbert damping, which is a prerequisite to keep a low STT-switching current. In principle, a fine tune of the irradiation conditions such as ion mass, energy and fluence may enable a more refined control of the easycone angle with a minimal decrease of the PMA. Nevertheless, the development of an irradiation technology of pMTJ will require a full understanding of the extent of the sample damage caused by the irradiation, especially its influence on the tunnel magnetoresistance. From a fundamental viewpoint, ion irradiation may be useful to study interface-induced magnetic phenomena, namely, the effect of the distribution of PMA and of the exchange coupling on the emergence of the second-order anisotropy.

\section{Conclusions}

We irradiated $\mathrm{MgO} / \mathrm{FeCoB}\left(\mathrm{t}_{\mathrm{FCB}}\right) / \mathrm{MgO}$ free layers, with and without a 0.2-nm-thick $\mathrm{W}$ or $\mathrm{Ta}$ spacer, and $t_{F C B}=3.0 \mathrm{~nm}$ or $t_{F C B}=2.6 \mathrm{~nm}$, with $400 \mathrm{keV} \mathrm{Ar}$ ions at fluences ranging from $10^{12} \mathrm{~cm}^{-}$

${ }^{2}$ to $10^{16} \mathrm{~cm}^{-2}$. The effective first-order PMA field, $B_{K_{1} 1}$, decreased linearly with the logarithm of the fluence, from $10^{12} \mathrm{~cm}^{-2}$ to $10^{15} \mathrm{~cm}^{-2}$. We suggest this decrease in $B_{k 1}$ to be a consequence of ioninduced intermixing at the $\mathrm{FeCoB} / \mathrm{MgO}$ interface.

We have shown that the decrease in PMA caused by the ion irradiation induces spin reorientations in these free layers. While the 3.0-nm-layer remains in its original easy-plane regime, we effectively produce easy-cone anisotropy, with different cone angles, in the thinner layer $(2.6 \mathrm{~nm})$ with a $\mathrm{W}$ spacer and obtain a complete spin reorientation from easy axis to easy cone and then to easy plane in the free layer with a Ta spacer. Importantly, the irradiation, at fluences for which these transitions occur, did not increase the Gilbert damping parameter, which is vital, from an application viewpoint, to keep a low current density for STT-switching.

\section{Acknowledgments}

B.M.S.T. and N.A.S. acknowledge financial support of the FCT of Portugal through Project No. I3N/FSCOSD (Reference No. FCT UID/CTM/50025/2013) and through the bursary 
PD/BD/113944/2015, as well as from the European project H2020-MSCA-RISE-2017-778308 SPINMULTIFILM. This study was also partially funded by ERC Adv. Grant MAGICAL No. 669204. A.A.T. acknowledges partial support of the Samsung Global MRAM Innovation Program, CEAEUROTALENTS fellowship. 


\section{References}

${ }^{1}$ B. Dieny and M. Chshiev, Rev. Mod. Phys. 89, 25008 (2017).

${ }^{2}$ H. Sato, M. Yamanouchi, S. Ikeda, S. Fukami, F. Matsukura, and H. Ohno, IEEE Trans. Magn. 49, 4437 (2013).

${ }^{3}$ H. Sato, E.C.I. Enobio, M. Yamanouchi, S. Ikeda, S. Fukami, S. Kanai, F. Matsukura, and H. Ohno, Appl. Phys. Lett. 105, 62403 (2014).

${ }^{4}$ E.C.I. Enobio, H. Sato, S. Fukami, F. Matsukura, and H. Ohno, IEEE Magn. Lett. 6, 5700303 (2015).

5 J.-H. Kim, J.-B. Lee, G.-G. An, S.-M. Yang, W.-S. Chung, H.-S. Park, and J.-P. Hong, Sci. Rep. 5, 16903 (2015).

${ }^{6}$ H. Almasi, M. Xu, Y. Xu, T. Newhouse-Illige, and W.G. Wang, Appl. Phys. Lett. 109, 32401 (2016).

7 S. Couet, T. Devolder, J. Swerts, S. Mertens, T. Lin, E. Liu, S. Van Elshocht, and G. Sankar Kar, Appl. Phys. Lett. 111, 152406 (2017).

8 D. Apalkov, B. Dieny, and J.M. Slaughter, Proc. IEEE 104, 685 (2016).

${ }^{9}$ P. Pirro, A. Hamadeh, M. Lavanant-Jambert, T. Meyer, B. Tao, E. Rosario, Y. Lu, M. Hehn, S. Mangin, and S. Petit Watelot, J. Magn. Magn. Mater. 432, 260 (2017).

${ }^{10}$ B.S. Tao, P. Barate, J. Frougier, P. Renucci, B. Xu, A. Djeffal, H. Jaffrès, J.M. George, X. Marie, S. Petit-Watelot, S. Mangin, X.F. Han, Z.G. Wang, and Y. Lu, Appl. Phys. Lett. 108, 152404 (2016).

${ }^{11}$ H. Arai, R. Matsumoto, S. Yuasa, and H. Imamura, Appl. Phys. Express 8, 83005 (2015).

12 N. Strelkov, A. Timopheev, R.C. Sousa, M. Chshiev, L.D. Buda-Prejbeanu, and B. Dieny, Phys. Rev. B 95, 184409 (2017).

${ }^{13}$ B.M.S. Teixeira, A.A. Timopheev, R. Schmidt, M.R. Soares, M. Seifert, V. Neu, and N.A. Sobolev, J. Phys. D: Appl. Phys. 49, 315002 (2016).

${ }^{14}$ A.A. Timopheev, R. Sousa, M. Chshiev, T. Nguyen, and B. Dieny, Sci. Rep. 6, 26877 (2016).

15 J.M. Shaw, H.T. Nembach, M. Weiler, T.J. Silva, M. Schoen, J.Z. Sun, D.C. Worledge, T.J. Watson, and Y. Heights, IEEE Trans. Magn. 6, 3500404 (2015).

${ }^{16}$ Y. Fu, I. Barsukov, J. Li, A.M. Gonçalves, C.C. Kuo, M. Farle, and I.N. Krivorotov, Appl. Phys. Lett. 108, 142403 (2016).

17 A.A. Timopheev, B.M.S. Teixeira, R.C. Sousa, S. Aufret, T.N. Nguyen, L.D. Buda-Prejbeanu, M. Chshiev, N.A. Sobolev, and B. Dieny, Phys. Rev. B 96, 14412 (2017).

${ }^{18}$ K.-W. Park, J.-Y. Park, S.C. Baek, D.-H. Kim, S.-M. Seo, S.-W. Chung, and B.-G. Park, Appl. Phys. Lett. 109, 12405 (2016). 
19 Y. Aleksandrov, C. Fowley, E. Kowalska, V. Sluka, O. YIldırlm, J. Lindner, B. Ocker, J. Fassbender, and A.M. Deac, AIP Adv. 6, 65321 (2016).

${ }^{20}$ B. Dieny and A. V Vedyayev, Europhys. Lett. 25, 723 (1994).

${ }^{21}$ J.Z. Sun, Phys. Rev. B 91, 174429 (2015).

${ }^{22}$ R. Matsumoto, H. Arai, S. Yuasa, and H. Imamura, Phys. Rev. B 92, 140409(R) (2015).

${ }^{23}$ J. Fassbender, D. Ravelosona, and Y. Samson, J. Phys. D: Appl. Phys. 37, R179 (2004).

${ }^{24}$ C. Chappert, H. Bernas, J. Ferré, V. Kottler, J.-P. Jamet, Y. Chen, E. Cambril, T. Devolder, F. Rousseaux, V. Mathet, and H. Launois, Science 280, 1919 (1998).

25 J. Ferré, T. Devolder, H. Bernas, J.-P. Jamet, V. Repain, M. Bauer, N. Vernier, and C. Chappert, J. Phys. D: Applied Phys. 36, 3103 (2003).

${ }^{26}$ C.T. Rettner, S. Anders, J.E.E. Baglin, T. Thomson, and B.D. Terris, Appl. Phys. Lett. 80, 279 (2002).

${ }^{27}$ V. Höink, M.D. Sacher, J. Schmalhorst, G. Reiss, D. Engel, D. Junk, and A. Ehresmann, Appl. Phys. Lett. 86, 152102 (2005).

${ }^{28}$ T. Devolder, I. Barisic, S. Eimer, K. Garcia, J.P. Adam, B. Ockert, and D. Ravelosona, J. Appl. Phys. 113, 203912 (2013).

${ }^{29}$ L. Herrera Diez, F. García-Sánchez, J.-P. Adam, T. Devolder, S. Eimer, M.S. El Hadri, A. Lamperti, R. Mantovan, B. Ocker, and D. Ravelosona, Appl. Phys. Lett. 107, 32401 (2015).

${ }^{30}$ L. Cuchet, R.C. Sousa, L. Vila, S. Auffret, B. Rodmacq, and B. Dieny, IEEE Trans. Magn. 50, 4401404 (2014).

${ }^{31}$ J.F. Ziegler, M.D. Ziegler, and J.P. Biersack, Nucl. Instr. Methods Phys. Res. B 268, 1818 (2010).

${ }^{32}$ S.M. Rezende, C. Chesman, M. A. Lucena, A. Azevedo, F.M. de Aguiar, and S.S.P. Parkin, J. Appl. Phys. 84, 958 (1998).

33 J.M.L. Beaujour, A.D. Kent, D. Ravelosona, I. Tudosa, and E.E. Fullerton, J. Appl. Phys. 109, 33917 (2011).

${ }^{34}$ S. Mizukami, Y. Ando, and T. Miyazaki, Jpn. J. Appl. Phys. 40, 580 (2001).

${ }^{35}$ X. Liu, W. Zhang, M.J. Carter, and G. Xiao, J. Appl. Phys. 110, 33910 (2011).

${ }^{36}$ S. lihama, S. Mizukami, H. Naganuma, M. Oogane, Y. Ando, and T. Miyazaki, Phys. Rev. B 89, 174416 (2014). 